Review began 01/06/2022 Review ended 01/12/2022 Published 01/17/2022

(c) Copyright 2022 Keramari et al. This is an open access article distributed under the terms of the Creative Commons Attribution License CCBY 4.0., which permits unrestricted use, distribution, and reproduction in any medium, provided the original author and source are credited.

\section{Clinical and Demographic Predictors of Health- Related Quality of Life After Orthopedic Surgery With Implant Placement}

Georgia Keramari $^{1}$, Ioannis Moisoglou ${ }^{2}$, Evangelia Meimeti ${ }^{3}$, Petros Galanis ${ }^{4}$, Evangelos C. Fradelos ${ }^{5}$ Ioanna V. Papathanasiou 6

1. Operating Room, General Hospital of Thessaloniki "George Papanikolaou", Thessaloniki, GRC 2. Pulmonary Clinic General Hospital of Lamia, Lamia, GRC 3. Training Coordination: Public Health/Community Nursing in Central and Western Macedonia, 3rd Regional Health Authority of Macedonia, Thessaloniki, GRC 4. Center for Health Services Management and Evaluation, Faculty of Nursing, National and Kapodistrian University of Athens, Athens, GRC 5. Nursing, University of Thessaly, Larissa, GRC 6. Community Nursing Lab, Faculty of Nursing, University of Thessaly, Larissa, GRC

Corresponding author: Ioannis Moisoglou, giannismois@gmail.com

\section{Abstract}

Background: Orthopedic surgeries can rehabilitate injuries and at the same time improve the patients' quality of life. The study aimed to assess patients' health-related quality of life (HRQOL) six months after an orthopedic surgery with implant placement.

Materials and methods: A cross-sectional study with the use of a structured questionnaire among 103 patients was conducted. The 36-Item Short Form Survey (SF-36) questionnaire was used to evaluate patients' quality of life.

Results: According to the findings of the multivariate linear regression analysis, low age, marital status (married in comparison to unmarried/ divorcees/widows), reduced intensity of the pain, and low educational attainment were associated with a better quality of life. Furthermore, the patients who were living with another person and the patients who underwent surgery on a part of the body other than the hip presented better quality of life. The results of the multivariate analysis explained 33\%-67\% of the variance of the SF-36 HRQOL.

Conclusion: Measuring quality of life is a valuable asset that helps to reveal the frail patient groups, in which health professionals will prioritize their care and the state in turn will design primary care services to meet their needs after discharge from the hospital.

Categories: Orthopedics

Keywords: orthopedic surgery, surgery, sf-36, orthopedic, health-related quality of life

\section{Introduction}

The health-related quality of life (HRQOL) measures the effect of a disease and its symptoms in patients' quality of life (QOL) [1]. The patient can be often found trapped in a vicious cycle where the disease's symptoms (causal indicators) can affect the patient's QOL. Through this negative effect, new problems arise (effect indicators) and turn into causal indicators that have a negative effect on the QOL [2]. Apart from the disease itself and its symptoms, there is also a significant number of individual and environmental characteristics that can have an impact on health outcomes and they should be examined during the studies on HRQOL [3].

According to the World Health Organization (WHO), musculoskeletal diseases are the first cause of disability worldwide. It is estimated that about $20 \%-33 \%$ of the total population suffers from a painful musculoskeletal condition $[4,5]$. The most common and disabling musculoskeletal condition is osteoarthritis, which often leads to orthopedic surgeries, back and neck pain, fractures associated with bone fragility, injuries, and systemic inflammatory conditions such as rheumatoid arthritis, whose rehabilitation and treatment require large amounts of money [5]. The aforementioned conditions affect the patients' QOL as well: they limit their daily activities, reduce their ability to work and participate in social roles, and have an impact on their mental wellbeing.

\section{Literature review}

Orthopedic surgeries can restore some of the damage and improve a patient's QOL, thus reversing the limitations and the pain the patient experiences. A prospective study in patients who underwent a hip and knee replacement surgery initially revealed a significant improvement in physical function and pain six months after the surgery, while at the end of the study (seven years follow-up) an improvement was 
reported in role physical and role emotional [6]. The fact that QOL remains high for years after the surgery is a very important finding and emphasizes the importance of such surgical procedures [7]. Patients who were prospectively observed for 24 months after a high tibial osteotomy showed a significant improvement in HRQOL and a decrease in pain during a six-month follow-up. Functional outcomes increased significantly in a 12-month follow-up and, at the final follow-up, $90 \%$ of the patients returned to their previous occupation without limitations [8].

However, several studies have shown that QOL does not always improve in patients who have undergone orthopedic surgery. Among the main factors that can have a negative effect on patients' QOL are sex, age, pain, and the body mass index (BMI). Data analysis on a large sample in the Netherlands showed that females and people with a higher BMI experienced some pain post-surgically. Advanced age and high BMI were related to lower QOL post-surgically, lower functionality, and more pain [9]. BMI is also associated with the average length of patients' stay at the hospital after orthopedic surgery, as obese patients required one more day of hospitalization and they presented a higher probability of readmission within 30 days after their discharge [10]. Patients with moderate to severe persistent pain have reported more limitations on their daily activities. Nevertheless, these limitations have not affected their QOL. On the contrary, severe pain and BMI were found to affect patients' daily functionality six months post-operatively [11].

The assessment of QOL and the investigation of the factors that can affect it have multiple benefits for the patient, the physician, and the health system. Studies revealed the factors and the vulnerable patient groups that require the attention of health-care professionals so that they will be able to provide integrated preoperative and post-operative care to cure the patients and improve their QOL. Regarding the health system, such studies can help the policymakers in decision-making about the design of health services that are suitable to meet the care needs of these patients in the community.

The study aimed to assess patients' HRQOL six months after an orthopedic surgery with implant placement.

\section{Materials And Methods \\ Study design}

A cross-sectional study using a structured questionnaire was conducted. The convenience sample consisted of patients who had undergone orthopedic surgery with implant placement in a Greek public general hospital. The questionnaire was completed after completion by patients' follow-up visit at the outpatients' orthopedic department, which is six months after their surgery. The study was conducted through phone interviews and lasted from March 20th to April 30th, 2020. A total of 103 patients were chosen to participate (convenience sampling). All patients agreed to participate (response rate 100\%). The inclusion criteria were the following: 18 or more years of age, ability to communicate in the Greek language, emergency, and scheduled surgeries. The operations were performed at the study hospital (Greek public general hospital). The patients, after the end of six months from the operation, were examined in the orthopedic outpatient clinic of the same hospital, in order to assess their post-operative condition.

\section{Ethics}

The study protocol was approved by the Ethical Committee of the participated hospital. The patients were approached by a member of the research team and asked if they wanted to participate in the study. Participants were informed about ethics issues before completing the questionnaires. In particular, they were informed that their participation is voluntary, anonymous, the collected data will be used exclusively for the purposes of the survey, and that they can leave the completion of the questionnaires at any time they wish. This study was conducted in compliance with the ethical standards of the responsible institution on human subjects as well as with the Helsinki Declaration.

\section{Study measures}

The 36-Item Short Form Survey (SF-36) [12], which assesses the QOL through 36 questions, was used to measure patients' QOL. The questionnaire had been translated and validated in Greek [13]. The questionnaire consists of eight sub-scales, which comprise two wider concise areas: physical and mental health. Cronbach's alpha of the Greek validated version of the instrument ranged from 0.79 (Social Functioning) to 0.95 (Role Physical), exceeding, in all cases, the 0.70 standard for group-level comparisons. A series of demographic and clinical characteristics such as age, gender, and marital status were also recorded.

\section{Statistical analysis}

Categorical variables are expressed as mean (n) and relative (\%) frequency, while quantitative variables are expressed as mean value, standard deviation, median, minimum value, and maximum value. The Kolmogorov-Smirnov test and graphs (histograms and normal Q-Q plots) were used to assess the normality of the distribution of the quantitative variables. The independent variables were the demographic and clinical characteristics, while the dependent variables were the scores on the SF-36. 


\section{Cureus}

The t-test (students' t-test) was used to assess the association between a quantitative variable and a binary variable, whereas the analysis of variance was used to assess the association between a quantitative variable and a qualitative variable with $>2$ categories. Pearson's correlation coefficient was used to assess the association between two quantitative variables. Spearman's correlation coefficient was used to assess the association between a quantitative variable that is normally distributed and a quantitative variable that is not normally distributed.

In the cases where $>2$ independent variables were statistically significant $(p<0.2)$ in bivariate analysis, a multivariate linear regression analysis with the scores as the dependent variables was applied. In this case, the backward stepwise linear regression was used. Regarding the multiple linear regression the coefficients' beta, the respective $95 \%$ confidence intervals, and p values are presented. In the case where the scores on the SF-36 did not follow a normal distribution, logarithms were used. The results are presented on a natural scale and not in the logarithmic scale to be simplified and comprehensible.

The two-sided level of statistical significance was set at 0.05 . Data analysis was performed using the IBM SPSS 21.0 (Statistical Package for Social Sciences; IBM Corp, Armonk, NY).

\section{Results}

The study population included 103 patients whose demographic characteristics are presented in Table 1 . The patients' mean age was 67.2 years, while the majority consisted of females (63.7\%), married (59.2\%), with children (80.6\%), primary school graduates (48\%), pensioners (64.1\%), insured (93.2\%), and lived with their spouse (55.9\%). 


\section{Cureus}

Characteristics

N (\%)

Sex

Male

Female

Age

Marital Status

Unmarried

Married

Divorcees

Widows

Number of Children

0

1

2

3

4

Educational Attainment

Uneducated

Primary School

Junior High School/High School

University

Employment Status

Employed

Unemployed

Pensioners

Cohabitation

Alone

Spouse

Children

Care Home

Parents

Insurance

Yes

No
$37(36.3)$

65 (63.7)

$67.2(14.5)^{*}$

$12(11.7)$

61 (59.2)

3 (2.9)

27 (26.2)

20 (19.4)

21 (20.4)

48 (46.6)

12 (11.7)

2 (1.9)

7 (6.9)

49 (48)

32 (31.4)

14 (13.7)

25 (24.3)

12 (11.7)

66 (64.1)

34 (33.3)

57 (55.9)

9 (8.8)

$1(1)$

1 (1)

96 (93.2)

7 (6.8)

TABLE 1: Demographic characteristics of the participants $(n=103)$.

*Mean (standard deviation)

The patients' clinical characteristics are presented in Table 2. The majority of them underwent hip surgery 


\section{Cureus}

(40.8\%); knee and hip surgeries were arthroplasty (joint replacement cases) and all other cases were fracture repair cases, and did physical therapy (80.6\%), the average of which was 13 . A percentage of $57.3 \%$ was experiencing pain and the average pain rating was 4.3 (on a scale from 0 to 10). A percentage of $24 \%$ were smokers and $43.7 \%$ had comorbidity. The most frequent comorbidities were hypertension, diabetes, coronary disease, cancer, and renal failure. Regarding the comorbidity score according to the Charlson Index [14], 56.3\% scored 0, 32\% scored 1, 9.7\% scored 2, $1 \%$ scored 3, and 1\% scored 4 . 


\section{Cureus}

Characteristic

N (\%)

Type of Surgery

Lower Leg

$12(11.7)$

Tibia/Fibula

$8(7.8)$

Knee

25 (24.3)

Hip

Arm/Shoulder

$42(40.8)$

$16(15.5)$

Physical Therapy (PT)

Yes

$83(80.6)$

No

Number of PT

20 (19.4)

$13(10.2)^{*}$

Second Surgery After Orthopedic

Yes

$5(5)$

No

Pain

Yes

No

Pain Intensity

Smoker

Yes

No

Comorbidity

Yes

No

Hypertension

Diabetes

Coronary Disease

Cancer

Renal Failure

Charlson Comorbidity Score Index

0

1

2

3

4

TABLE 2: Patients' clinical characteristics $(n=103)$.
96 (95)

59 (57.3)

44 (42.7)

$4.3(1.7)^{*}$

$24(24)$

$76(76)$

45 (43.7)

58 (56.3)

34 (33)

$21(20.4)$

8 (7.8)

4 (3.9)

4 (3.9)

58 (56.3)

$33(32)$

$10(9.7)$

1 (1)

$1(1)$

*Mean (standard deviation). 


\section{Cureus}

The internal consistency coefficients (Cronbach's alpha) for the eight subscales of SF-36 are presented in Table 3. The minimum acceptable value for Cronbach's alpha internal consistency coefficient was $>0.7$. In all scales, alpha was $>0.70$, which shows a very good internal consistency.

\begin{tabular}{|l|l|}
\hline Scale & Cronbach's Alpha \\
\hline Physical Function & 0.92 \\
Role Physical & 0.70 \\
Bodily Pain & 0.74 \\
General Health & 0.83 \\
Vitality & 0.82 \\
Social Functioning & 0.72 \\
Role Emotional & 0.76 \\
Mental Health & 0.85 \\
Overall & 0.91 \\
\hline
\end{tabular}

\section{TABLE 3: Cronbach's alpha for the scales and overall SF-36.}

The descriptive results for the scores in the SF-36 are presented in Table 4. Both the physical health component summary and the mental health component summary were lower than 50 , which shows an inferior QOL in comparison to the general population. Moreover, the mental health component summary scored higher than the physical health component summary, which shows that the patients' mental health was better than their physical health.

\begin{tabular}{|c|c|c|c|c|c|}
\hline Scale & Mean & Standard Deviation & Median & Minimum Value & Maximum Value \\
\hline Physical Function & 45.8 & 32.1 & 45 & 0 & 100 \\
\hline Role Physical & 42 & 46.3 & 0 & 0 & 100 \\
\hline Bodily Pain & 67.9 & 27.9 & 74 & 0 & 100 \\
\hline General Health & 55.8 & 28.3 & 60 & 0 & 100 \\
\hline Vitality & 56.7 & 28.3 & 60 & 0 & 100 \\
\hline Social Functioning & 71.2 & 31.7 & 75 & 0 & 100 \\
\hline Role Emotional & 48.9 & 45.9 & 33.3 & 0 & 100 \\
\hline Mental Health & 65.1 & 26.9 & 72 & 4 & 100 \\
\hline General Well-being & 38.8 & 11.5 & 40 & 17 & 58.6 \\
\hline General Mental Health & 46.9 & 13.1 & 48.9 & 15 & 65 \\
\hline
\end{tabular}

TABLE 4: Descriptive statistics for the scores on the SF-36 subscales.

SF-36, 36-Item Short Form Survey.

Bivariate analysis between the demographic and clinical characteristics using the scores of the eight subscales of the questionnaire was conducted. Following the bivariate analysis, multivariate linear regression was applied to the independent variables that presented an association of $0.20(p<0.20)$. The results are presented in Table 5. According to the findings of the multivariate linear regression analysis, low age, marital status (married in comparison to unmarried/divorcees/widows), reduced intensity of the pain, and low educational attainment were associated with a better QOL. Also, the patients who lived with another 


\section{Cureus}

person and the patients who underwent surgery on a part of the body other than the hip presented a better QOL. The results of the multivariate analysis explained 33\%-67\% of the variance of the SF-36 HRQOL

\begin{tabular}{|c|c|c|c|c|}
\hline Dependent and Independent Variables & Coefficient Beta & 95\% Confidence interval for Beta & p-Value & Adjusted $\mathbf{R}^{2}$ \\
\hline General Well-being & & & & $66 \%$ \\
\hline Age & -0.4 & -0.6 to -0.2 & 0.001 & \\
\hline Married vs Unmarried/Divorcees/Widows & 5.2 & 1.6 to 8.8 & 0.006 & \\
\hline Pain intensity & -1.9 & -3 to -0.9 & 0.001 & \\
\hline Other Operated Body Members vs The Hip & 5.4 & 1.6 to 9.2 & 0.006 & \\
\hline General Mental Health & & & & $45 \%$ \\
\hline Age & 0.3 & -0.5 to -0.04 & 0.021 & \\
\hline Married vs Unmarried/Divorcees/Widows & 7.5 & 2.1 to 12.9 & 0.008 & \\
\hline Other Operated Body Members vs The Hip & 9.5 & 3.9 to 15.3 & 0.001 & \\
\hline Physical Function & & & & $62 \%$ \\
\hline Age & 0.7 & 1.1 to 0.3 & 0.001 & \\
\hline Married vs Unmarried/Divorcees/Widows & 14.5 & 3.9 to 25.1 & 0.008 & \\
\hline Pain intensity & 3.6 & 6.7 to 0.6 & 0.021 & \\
\hline Other Operated Body Members vs The Hip & 21.1 & 10.1 to 32.1 & 0.001 & \\
\hline Role Physical & & & & $33 \%$ \\
\hline Age & -1.4 & -2.5 to -0.3 & 0.016 & \\
\hline Married vs Unmarried/Divorcees/Widows & 29 & 9.5 to 48.5 & 0.004 & \\
\hline Educational Attainment & -20 & -36 to -3 & 0.023 & \\
\hline Bodily Pain & & & & $56 \%$ \\
\hline Married vs Unmarried/Divorcees/Widows & 9.5 & 0.7 to 18.3 & 0.040 & \\
\hline Pain intensity & -5.8 & -8.3 to -3.2 & 0.001 & \\
\hline Other Operated Body Members vs The Hip & 17.6 & 9.1 to 26.1 & 0.001 & \\
\hline General Health & & & & $67 \%$ \\
\hline Age & 0.9 & -1.3 to -0.6 & 0.001 & \\
\hline Married-Unmarried/Divorcees/Widows & 13 & 3.9 to 22.1 & 0.006 & \\
\hline Pain intensity & -3.9 & -6.5 to -1.2 & 0.005 & \\
\hline Other Operated Body Members vs The Hip & 11.9 & 2.4 to 21.3 & 0.015 & \\
\hline Vitality & & & & $55 \%$ \\
\hline Age & -1.1 & -1.7 to -0.5 & 0.001 & \\
\hline Married vs Unmarried/Divorcees/Widows & 13.5 & 2.8 to 24.2 & 0.015 & \\
\hline Other operated body members vs the hip & 12.9 & 1.5 to 24.1 & 0.027 & \\
\hline Social Functioning & & & & $56 \%$ \\
\hline Age & -2.1 & -3.1 to -1 & 0.001 & \\
\hline Married vs Unmarried/Divorcees/Widows & 23.8 & 3.8 to 43.7 & 0.021 & \\
\hline Other Operated Body Members vs The Hip & 25 & 11.5 to 38.7 & 0.001 & \\
\hline Role Emotional & & & & $34 \%$ \\
\hline
\end{tabular}




\section{Cureus}

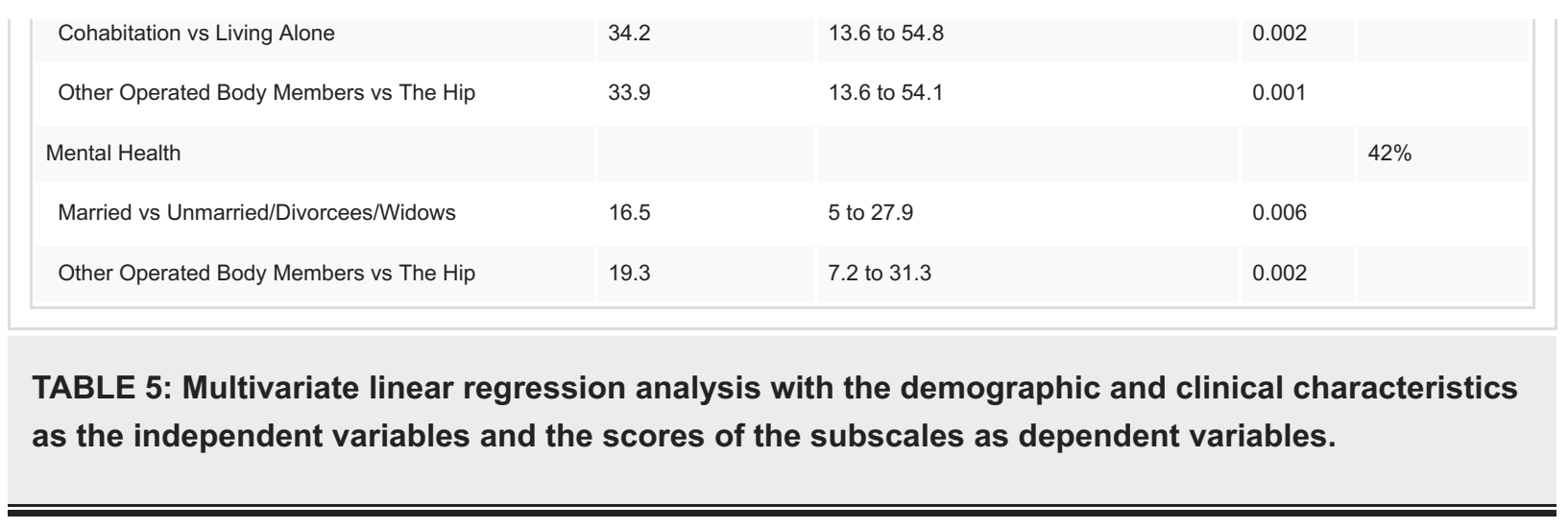

\section{Discussion}

The present study investigated patients' QOL six months after an orthopedic surgery with implant placement. According to the results of the study, both the physical health component summary and the mental health component summary were lower than 50, which shows an inferior QOL in comparison to the general population. These findings can be interpreted by the fact that often patients after orthopedic surgery need time to achieve a restoration of their QOL, and often a longer period of six months is required, which can reach years later [6,7]. Also, as most of the participants report the existence of pain (with an average pain intensity value of 4.3 ), are elderly, and suffer from comorbidities, all of these may create significant limitations in their daily lives with an impact on their QOL. Regarding the participants' demographic characteristics, the young, the married, and those who lived with their partner were found to have a better QOL and presented better results in all the scales of the questionnaire. A person's ability to perform his daily family and social activities demands good physical health and good physical function, to a great extent. The support and the help that other family members can provide can contribute to a more effective fulfillment of these efforts. Patients who live alone present more functional limitations after orthopedic surgery [15]. Also, the post-operative physical function, social function, and vitality are affected by the patients' age; older people experience greater impairment of their QOL associated with the above scales [16]. The benefits associated with marital status do not affect only the orthopedic patients. They can have a positive effect on the survival of oncological patients as well [17]. According to a recent study in the population of Sweden, married people have up to 2 times lower probability of mortality of COVID-19 in comparison to unmarried people or people who live alone [18]. The implementation of interdisciplinary exercise schedules in the community to improve the strength of the elderly has proven effective, as it can improve the patients' pain level, physical function, role physical, vitality, social networking, and mental health [19-21], supported by the regions or municipalities, have also proven beneficial for the elderly or lonesome people of the community. Such programs offer various primary care services such as personal help and adult day-services, which help people with disabilities live in the community [22]. Many municipalities in Greece implement a similar program called "In-House Help”. This primary care program provides help and care to the elderly and chronic patients who participate in order to cover their physical, mental, and sentimental needs. Several studies have proven the program's efficiency in covering the aforementioned needs of the participants $[23,24]$.

According to the clinical characteristics in the present study, patients who underwent surgery on the hip and experienced post-operative pain of high intensity presented an inferior QOL. High-intensity post-operative pain is common in orthopedic surgeries and can reduce patients' functionality and health level, affect their QOL, and can even result in mortality $[25,26]$. The present study's findings partially coincide with the respective findings of a study on total hip replacements, where a total of 104 patients participated six months after their surgery. A percentage between $26 \%$ and $58 \%$ admitted that they experienced persistent pain on the hip [11]. The patients who experienced moderate to severe pain also reported more limitations on their daily activities. However, those limitations were not found to affect the patients' QOL.

A hip replacement surgery can cause severe mobility difficulties, which explains why patients who underwent surgical procedures other than hip replacement reported a better QOL in the present study. Regardless of the good function of other body members, mobility limitations also impede any activity. The limitations of hip surgery are more determinant in comparison to the limitations of surgical procedures in other parts of the lower extremities [27]. The improvement of the patients' functionality, hence the improvement of their QOL after a hip replacement, can include threefold interventions. The first one refers to the reduction of waiting for surgery; waiting affects QOL, thus affecting the post-surgical outcome [28]. The other two interventions refer to the interdisciplinary cooperation for the patient's optimum care, which does not only include the orthopedics specialist. The cooperation of health specialists (psychologists, physical therapists, nutritionists), which should begin when the patient is admitted to the hospital and continue even after the patient has returned home, has been found to have beneficial results [29]. The benefits of the interdisciplinary interventions are important for the hospitals and the health-care system as well, as they can help reduce the average hospitalization period and the cost of care [30]. 


\section{Limitations}

The current study contains a series of limitations that need to be assessed when generalizing the results. The study was conducted in only one hospital, with the participation of a relatively small sample of patients. Also, as the questionnaires were answered through phone interviews, it was impossible to estimate the patients' BMI, which according to the literature can affect the patients' QOL. Also, the comorbidity score has been estimated using the patients' reports on chronic illnesses, and not their medical files. As a result, relevant information might have been omitted.

\section{Conclusions}

The present study on patients who have undergone orthopedic surgery with implant placement has revealed a series of demographic and clinical data, which have revealed the frail patient groups and the areas where health-care professionals and the state should focus. In addition, future studies should have long-term evaluation points (follow-up 1-3 years) in order to reliably capture the value of interventions in improving the QOL of the patients, as after a period of six months, there is likely to be a small improvement in QOL. It is important to measure QOL to better examine these areas, which have a multidimensional role and can affect not only the QOL but the patients' mortality as well. The benefits from the improvement of the QOL can expand to the health-care systems, thus reducing the required resources for patients' care.

\section{Additional Information \\ Disclosures}

Human subjects: Consent was obtained or waived by all participants in this study. Ethics Committee of General Hospital of Thessaloniki “George Papanikolaou” issued approval 5.18.03.2020. The study was approved by the Ethics Committee of General Hospital of Thessaloniki "George Papanikolaou". Animal subjects: All authors have confirmed that this study did not involve animal subjects or tissue. Conflicts of interest: In compliance with the ICMJE uniform disclosure form, all authors declare the following: Payment/services info: All authors have declared that no financial support was received from any organization for the submitted work. Financial relationships: All authors have declared that they have no financial relationships at present or within the previous three years with any organizations that might have an interest in the submitted work. Other relationships: All authors have declared that there are no other relationships or activities that could appear to have influenced the submitted work.

\section{References}

1. Mao Z, Ahmed S, Graham C, Kind P, Sun YN, Yu CH: Similarities and differences in health-related qualityof-life concepts between the east and the west: a qualitative analysis of the content of health-related quality-of-life measures. Value Health Reg Issues. 2021, 24:96-106. 10.1016/j.vhri.2020.11.007

2. Fayers PM, Hand DJ, Bjordal K, Groenvold M: Causal indicators in quality of life research . Qual Life Res. 1997, 6:393-406. 10.1023/A:1018491512095

3. Estoque RC, Togawa T, Ooba M, Gomi K, Nakamura S, Hijioka Y, Kameyama Y: A review of quality of life (QOL) assessments and indicators: towards a "QOL-Climate" assessment framework. Ambio. 2019, 48:61938. 10.1007/s13280-018-1090-3

4. Brennan-Olsen SL, Cook S, Leech MT, et al.: Prevalence of arthritis according to age, sex and socioeconomic status in six low and middle income countries: analysis of data from the World Health Organization study on global AGEing and adult health (SAGE) Wave 1. BMC Musculoskelet Disord. 2017, 18:271. 10.1186/s12891-017-1624-z

5. World Health Organization. Musculoskeletal Conditions. (2021). Accessed: December 16, 2021: https://www.who.int/news-room/fact-sheets/detail/musculoskeletal-conditions.

6. Bruyère $O$, Ethgen $O$, Neuprez A, Zégels B, Gillet P, Huskin JP, Reginster JY: Health-related quality of life after total knee or hip replacement for osteoarthritis: a 7-year prospective study. Arch Orthop Trauma Surg. 2012, 132:1583-7. 10.1007/s00402-012-1583-7

7. Neuprez A, Neuprez AH, Kaux JF, et al.: Total joint replacement improves pain, functional quality of life, and health utilities in patients with late-stage knee and hip osteoarthritis for up to 5 years. Clin Rheumatol. 2020, 39:861-71. 10.1007/s10067-019-04811-y

8. Saier T, Minzlaff P, Feucht MJ, et al.: Health-related quality of life after open-wedge high tibial osteotomy . Knee Surg Sports Traumatol Arthrosc. 2017, 25:934-42. 10.1007/s00167-015-3938-4

9. Hofstede SN, Gademan MG, Stijnen T, Nelissen RG, Marang-van de Mheen PJ: The influence of preoperative determinants on quality of life, functioning and pain after total knee and hip replacement: a pooled analysis of Dutch cohorts. BMC Musculoskelet Disord. 2018, 19:68. 10.1186/s12891-018-1991-0

10. Chen JY, Lo NN, Chong HC, et al.: The influence of body mass index on functional outcome and quality of life after total knee arthroplasty. Bone Joint J. 2016, 98-B:780-5. 10.1302/0301-620X.98B6.35709

11. Erlenwein J, Müller M, Falla D, et al.: Clinical relevance of persistent postoperative pain after total hip replacement - a prospective observational cohort study. J Pain Res. 2017, 10:2183-93. 10.2147/JPR.S137892

12. Ware JE Jr, Sherbourne CD: The MOS 36-Item Short-Form Health Survey (SF- 36): I. Conceptual framework and item selection. Med Care. 1992, 30:473-83. 10.2307/3765916

13. Pappa E, Kontodimopoulos N, Niakas D: Validating and norming of the Greek SF-36 Health Survey . Qual Life Res. 2005, 14:1433-8. 10.1007/s11136-004-6014-y

14. Charlson ME, Pompei P, Ales KL, MacKenzie CR: A new method of classifying prognostic comorbidity in longitudinal studies: development and validation. J Chronic Dis. 1987, 40:373-83. 10.1016/00219681(87)90171-8 
15. Desmeules F, Dionne CE, Belzile ÉL, Bourbonnais R, Champagne F, Frémont P: Determinants of pain, functional limitations and health-related quality of life six months after total knee arthroplasty: results from a prospective cohort study. BMC Sports Sci Med Rehabil. 2013, 5:2. 10.1186/2052-1847-5-2

16. Alentorn-Geli E, Leal-Blanquet J, Guirro P, Hinarejos P, Pelfort X, Puig-Verdié L: Comparison of quality of life between elderly patients undergoing TKA. Orthopedics. 2013, 36:e415-9. 10.3928/01477447-2013032715

17. Aizer AA, Chen MH, McCarthy EP, et al.: Marital status and survival in patients with cancer . J Clin Oncol. 2013, 31:3869-76. 10.1200/JCO.2013.49.6489

18. Drefahl S, Wallace M, Mussino E, et al.: A population-based cohort study of socio-demographic risk factors for COVID-19 deaths in Sweden. Nat Commun. 2020, 11:5097. 10.1038/s41467-020-18926-3

19. Tarazona-Santabalbina FJ, Gómez-Cabrera MC, Pérez-Ros P, et al.: A multicomponent exercise intervention that reverses frailty and improves cognition, emotion, and social networking in the community-dwelling frail elderly: a randomized clinical trial. J Am Med Dir Assoc. 2016, 17:426-33. 10.1016/j.jamda.2016.01.019

20. Ettinger WH, Burns R, Messier SP, et al.: A randomized trial comparing aerobic exercise and resistance exercise with a health education program in older adults with knee osteoarthritis. The Fitness Arthritis and Seniors Trial (FAST). JAMA. 1997, 277:25-31. 10.1001/jama.1997.03540250033028

21. Shyu YI, Liang J, Wu CC, Su JY, Cheng HS, Chou SW, Yang CT: A pilot investigation of the short-term effects of an interdisciplinary intervention program on elderly patients with hip fracture in Taiwan. J Am Geriatr Soc. 2005, 53:811-8. 10.1111/j.1532-5415.2005.53253.x

22. Muramatsu N, Yin H, Hedeker D: Functional declines, social support, and mental health in the elderly: does living in a state supportive of home and community-based services make a difference?. Soc Sci Med. 2010, 70:1050-8. 10.1016/j.socscimed.2009.12.005

23. Frengidou E, Frengidou E, Nikolentzos A, Galanis P, Papadopoulou A: Investigating user satisfaction with the services provided by the care program "Helping Elderly at Home" and assessing their self-evaluation health level: the case of the program "Helping Elderly at Home” in the municipality of Kilkis. In Greek. Rostrum Asclepius. 2019, 18:377. 10.5281/zenodo.3469096

24. Alexias G, Flamou A: Evaluation of the social and psycho-emotional support of aged people facing health and social isolation and exclusion problems: the "in house-help" program. Arch Hell Med. 2007, 24:37-42.

25. Alexiou KI, Roushias A, Varitimidis SE, Malizos KN: Quality of life and psychological consequences in elderly patients after a hip fracture: a review. Clin Interv Aging. 2018, 13:143-50. 10.2147/CIA.S150067

26. Wylde V, Blom AW, Whitehouse SL, Taylor AH, Pattison GT, Bannister GC: Patient-reported outcomes after total hip and knee arthroplasty: comparison of midterm results. J Arthroplasty. 2009, 24:210-6. 10.1016/j.arth.2007.12.001

27. Orive M, Aguirre U, García-Gutiérrez S, et al.: Changes in health-related quality of life and activities of daily living after hip fracture because of a fall in elderly patients: a prospective cohort study. Int J Clin Pract. 2015, 69:491-500. 10.1111/ijcp.12527

28. Ackerman IN, Bennell KL, Osborne RH: Decline in health-related quality of life reported by more than half of those waiting for joint replacement surgery: a prospective cohort study. BMC Musculoskelet Disord. 2011, 12:108. 10.1186/1471-2474-12-108

29. Peeters CM, Visser E, Van de Ree CL, Gosens T, Den Oudsten BL, De Vries J: Quality of life after hip fracture in the elderly: a systematic literature review. Injury. 2016, 47:1369-82. 10.1016/j.injury.2016.04.018

30. Swart E, Vasudeva E, Makhni EC, Macaulay W, Bozic KJ: Dedicated perioperative hip fracture comanagement programs are cost-effective in high-volume centers: an economic analysis. Clin Orthop Relat Res. 2016, 474:222-33. 10.1007/s11999-015-4494-4 